\title{
Respiratory Oscillations and Heat Evolution in Synchronous Cultures of Candida utilis
}

\author{
By JALIL KADER* AND DAVID LLOYD \\ Department of Microbiology, University College, Newport Road, Cardiff CF2 1 TA
}

(Received 26 January 1979)

\begin{abstract}
Synchronous cultures of the budding yeast Candida utilis prepared by continuous-flow size selection showed respiratory oscillations when the energy source was either glucose, acetate or glycerol. The period of the oscillations was about one-third of the cell cycle time (i.e. about $0.5 \mathrm{~h}$ ). No fluctuations in heat evolution could be detected. In organisms growing with acetate or glycerol, the effects of cyanide, $N, N^{\prime}$-dicyclohexylcarbodi-imide and carbonyl cyanide $m$-chlorophenylhydrazone (maximum inhibition of respiration at respiratory maxima, maximum uncoupling of energy conservation at respiratory minima) suggest that the control mechanism responsible for the oscillations is mitochondrial respiratory control in vivo. The effects of cyanide and $N, N^{\prime}$-dicyclohexylcarbodi-imide on the respiration of cultures growing synchronously with glucose were different from those for cultures growing with the non-fermentable substrates; this suggests that the mitochondrial respiratory system interacts with the early reactions of glucose utilization.
\end{abstract}

\section{INTRODUCTION}

Changes in oxygen consumption rates during the growth of synchronous cultures of eukaryotic micro-organisms provide insights into mechanisms of development and control of mitochondrial activities (see Lloyd, 1974). It is essential in such studies to prepare synchronously dividing populations by a procedure which does not disturb their energy metabolism; synchrony with minimal perturbation of respiratory rates and adenine nucleotide pools can be achieved using either the continuous-flow selection method (Lloyd et al., 1975) or, in the case of Acanthamoeba castellanii, by a low force-time centrifugal field in tubes (Chagla \& Griffiths, 1978). In these synchronously dividing populations the rate of respiration was found to oscillate with a period of about $1 \mathrm{~h}$ in both Tetrahymena pyriformis (Lloyd et al., 1978) and A. castellanii (Edwards \& Lloyd, 1978), when the protozoa were growing in complex media. In the present investigation we have studied the development of respiration in continuous-flow selected synchronous cultures of Candida utilis growing in chemically defined media with either glucose, glycerol or acetate as sole source of carbon and energy for growth. Although the periodicity of the respiratory oscillations is similar with different carbon sources, the effects of $\mathrm{KCN}$ and of $N, N^{\prime}$-dicyclohexylcarbodi-imide (inhibitors of electron transport and energy transfer, respectively) are different on cells growing with glucose from those observed for organisms growing with the non-fermentable carbon sources.

\section{METHODS}

Abbreviations. CCCP, Carbonyl cyanide $m$-chlorophenylhydrazone; DCCD, $N, N^{\prime}$-dicyclohexylcarbodiimide.

Maintenance and growth of the organism. Candida utilis NCYC 193 was maintained on $10 \%(\mathrm{w} / \mathrm{v})$ glucose/ malt extract/agar slopes at $2{ }^{\circ} \mathrm{C}$. Stock cultures were subcultured monthly and maintained at $2{ }^{\circ} \mathrm{C}$.

* Present address: Unit Mikrobiologi, Universiti Kebangsaan Malaysia, Kuala Lumpur, Malaysia. 
The chemically defined media contained (per litre): $10 \mathrm{~g}$ glucose or $12.2 \mathrm{~g}$ glycerol or $3.8 \mathrm{~g}$ sodium acetate (anhydrous), $5 \mathrm{~g}\left(\mathrm{NH}_{4}\right)_{2} \mathrm{SO}_{4}, 2 \mathrm{~g} \mathrm{NaH}_{2} \mathrm{PO}_{4} .2 \mathrm{H}_{2} \mathrm{O}, 100 \mathrm{ml}$ salts solution, $1.0 \mathrm{ml}$ trace elements solution and $0.25 \mathrm{mg}$ biotin. The salts solution contained (per litre): $10 \mathrm{~g} \mathrm{KCl}, 10.8 \mathrm{~g} \mathrm{MgCl}_{2}, 132 \mathrm{mg} \mathrm{CaCl}_{2} .2 \mathrm{H}_{2} \mathrm{O}$ and

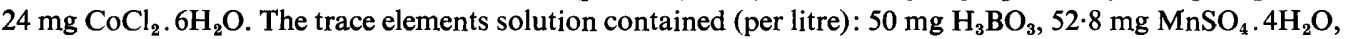
$40 \mathrm{mg} \mathrm{ZnSO}_{4} .7 \mathrm{H}_{2} \mathrm{O}, 20 \mathrm{mg} \mathrm{FeCl}_{3}, 16 \mathrm{mg} \mathrm{H} \mathrm{MoO}_{4} . \mathrm{H}_{2} \mathrm{O}, 10 \mathrm{mg} \mathrm{KI}, 4 \mathrm{mg} \mathrm{CuSO} \cdot 5 \mathrm{H}_{2} \mathrm{O}$ and $100 \mathrm{mg}$ citric acid. $\mathrm{NaH}_{2} \mathrm{PO}_{4}$ and glucose solutions were autoclaved separately, and biotin was sterilized by filtration.

Starter cultures $(250 \mathrm{ml}$ in 11 conical flasks) were inoculated from stock cultures and shaken at $75 \mathrm{rev}$. $\mathrm{min}^{-1}$ on a rotary shaker at $30^{\circ} \mathrm{C}$. Batch cultures (81) were grown in a 141 Microferm Fermentor (New Brunswick Scientific Co.) at $30^{\circ} \mathrm{C}$, with aeration at 11 air $\mathrm{min}^{-1}$ (l culture $)^{-1}$ and stirring at $400 \mathrm{rev} . \mathrm{min}^{-1}$. Organisms were counted following the criteria of Williamson \& Scopes (1960) in a Thoma haemocytometer slide (Hawksley, Lancing, Sussex) after suitable dilution. A particle with a single bud was scored as one cell, and a particle containing two or three buds was counted as two cells.

Preparation of synchronous cultures. Synchronous cultures were prepared by the continuous-flow selection method of Lloyd et al. (1975), using a flow rate of $420 \mathrm{ml} \mathrm{min}{ }^{-1}$ and a rotor speed of about $3500 \mathrm{rev} . \mathrm{min}^{-1}$. Small adjustments were made to the rotor speed until exactly $10 \%$ of the original population escaped harvesting in the rotor before collection of the selected population was begun.

Assessment of synchrony. The degree of synchrony was assessed by the synchrony index $(F)$ of Elumenthal \& Zahler (1962), calculated from the equation:

$$
F=\left(N / N_{0}\right)-2^{t / g}
$$

where $F$ has a maximum value of 1.0 in a culture exhibiting theoretically perfect synchrony, $N$ is the number of organisms at time $t, N_{0}$ is the number of organisms at zero time and $g$ is the mean generation time. In the presentation of results, vertical lines indicate the mid-points of doublings in cell numbers, and $F_{1}$ and $F_{2}$ denote the synchrony indices of the first and second doublings in yeast numbers, respectively.

Measurement of oxygen uptake. Samples were removed at $10 \mathrm{~min}$ intervals during growth and oxygen uptake was measured polarographically (Lloyd \& Brookman, 1967). KCN was used as an aqueous solution $(0.01 \mathrm{M})$. DCCD $\left(10 \mathrm{mg} \mathrm{ml}^{-1}\right)$ was dissolved in $10 \%(\mathrm{v} / \mathrm{v})$ dimethylformamide, as was CCCP $\left(0 \cdot 2 \mathrm{mg} \mathrm{ml}^{-1}\right)$.

Measurements of heat evolution. Measurements were made as described by Poole et al. (1973). The culture was pumped from the culture vessel at a constant rate of $54 \mathrm{ml} \mathrm{h}^{-1}$ to a T-piece, where it was mixed with a constant flow of air $\left(54 \mathrm{ml} \mathrm{h}^{-1}\right)$. The temperature of the thermostatic air bath was $30^{\circ} \mathrm{C}$; water at $15^{\circ} \mathrm{C}$ was used to assist dissipation of heat from the instrument. The instrument was calibrated at the end of the experiment, with aerated medium flowing through the cell, by applying an internal current of 1.0 or $1.5 \mathrm{~mA}$ for 800 or $1000 \mathrm{~s}$ to achieve a steady state. The calibration was recorded in $\mu \mathrm{J} \mathrm{s}{ }^{-1}$.

Chemicals. KCN and DCCD were from BDH; CCCP was from Calbiochem.

\section{RESULTS}

\section{Respiration during growth of asynchronous cultures}

In asynchronous cultures growing on glucose, acetate or glycerol as sole carbon sources, cell numbers and respiration rates increased exponentially, with no detectable sign of perturbation even over the first 30 min after resuspension (Fig. 1). The maximum deviation from the best-fit line on the respiration plot was $3 \cdot 3 \%$. Mean generation times for cultures containing glucose, acetate or glycerol were 96,122 and $105 \mathrm{~min}$, respectively; corresponding times for doubling of oxygen consumption rates were 90,96 and $105 \mathrm{~min}$. The respiration of cultures growing exponentially with glucose or glycerol was $50 \%$ inhibited by $50 \mu \mathrm{M}$ $\mathrm{KCN}$, whereas only $30 \mu \mathrm{M}-\mathrm{KCN}$ was required for a similar degree of inhibition in acetatecontaining cultures. Complete inhibition of respiration of cultures growing on glucose, glycerol or acetate was produced by $150 \mu \mathrm{M}-\mathrm{KCN}$. Concentrations of DCCD giving $50 \%$ inhibition were 75,80 and $30 \mu \mathrm{M}$ for cultures containing glucose, glycerol or acetate, respectively. Maximum uncoupling of respiration of all three types of culture was observed with $3 \mu \mathrm{M}-\mathrm{CCCP}$.

\section{Respiration of synchronous cultures and the effects of inhibitors and an uncoupler}

The pattern of increase in respiration in a culture growing synchronously with glucose is shown in Fig. 2. The first mid-point of increase in cell numbers occurred after $94 \mathrm{~min}$, and an exact doubling was observed after $130 \mathrm{~min}$. Synchrony indices for the first and 


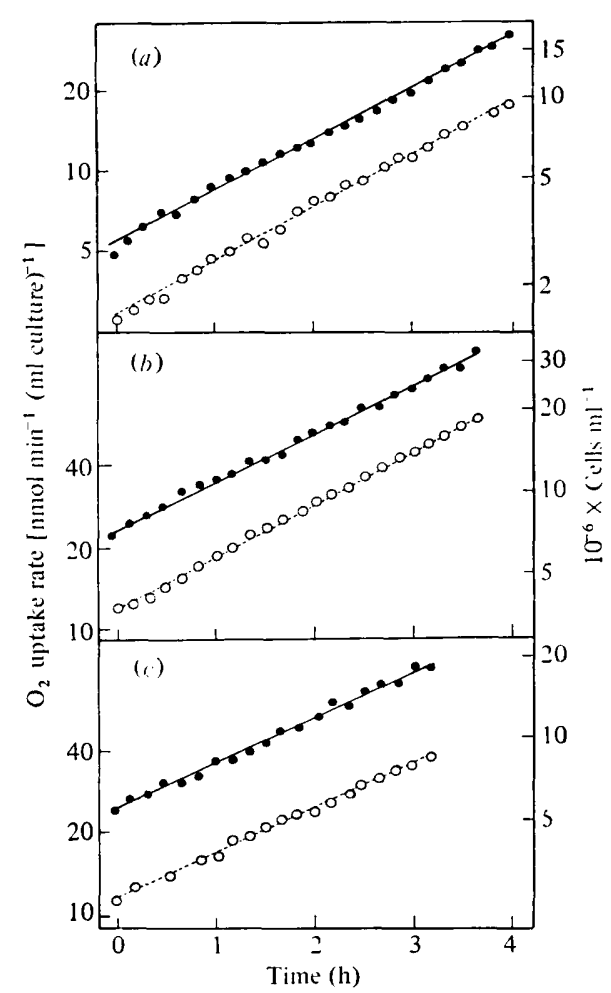

Fig. 1

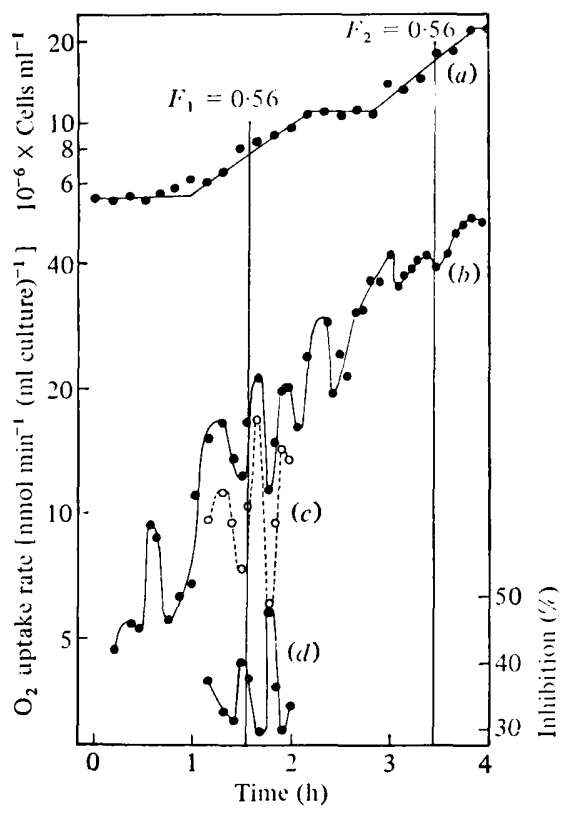

Fig. 2

Fig. 1. Changes in cell numbers $(\odot)$ and respiration rates $(\bigcirc)$ in asynchronous cultures of $C$. utilis growing with glucose $(a)$, acetate $(b)$ and glycerol $(c)$ as sole carbon sources. At time zero, each culture was resuspended after centrifugation at $2000 \mathrm{~g}$ for $2 \mathrm{~min}$ at $30^{\circ} \mathrm{C}$.

Fig. 2. Effects of cyanide on respiratory oscillations in a synchronously dividing culture of $C$. utilis with glucose as carbon source. The synchronous culture initially contained $10 \%$ of the original population of the exponentially growing culture and was concentrated fourfold by centrifugation at $2000 \mathrm{~g}$ for $2 \mathrm{~min}$ followed by resuspension in conditioned growth medium. At intervals, samples were withdrawn from the culture and rates of respiration were determined in the presence or absence of $\mathrm{KCN}$. (a) Cell numbers; (b) respiration rate in the absence of $\mathrm{KCN}$; $(c)$ respiration rate in the presence of $10 \mu \mathrm{M}-\mathrm{KCN}$; $(d)$ inhibition of respiration $(\%)$ resulting from addition of $\mathrm{KCN}$.

second cycles were identical. The rate of respiration of the culture increased exponentially overall, but an oscillation superimposed upon this baseline showed a peak to trough amplitude of between 30 and $60 \%$ of the minimum respiration rates. The average periodicity was $26 \mathrm{~min}$. Addition of $10 \mu \mathrm{M}-\mathrm{KCN}$ to samples withdrawn from the culture at respiratory minima gave almost $50 \%$ inhibition of oxygen uptake rates, whereas this concentration of $\mathrm{KCN}$ gave only about $30 \%$ inhibition of respiration in samples respiring maximally.

DCCD $(75 \mu \mathrm{M})$, an inhibitor of energy transfer at the level of the ATP synthetase reaction (Kováč et al., 1968), also showed a differential effect depending on whether samples were tested at respiratory maxima or minima (Fig. 3). As with cyanide, sensitivity to inhibition was most marked when respiratory rates were at their minimum values.

Maximum stimulation of the respiration of cultures growing synchronously with glucose by the uncoupling agent CCCP $(3 \mu \mathrm{M})$ was observed when this compound was added at respiratory minima. At these times over $60 \%$ acceleration of oxygen consumption rates was observed, in contrast to the small extent of uncoupling (20 to $30 \%$ stimulation of respiration) observed when $\mathrm{CCCP}$ was added at respiratory maxima. A similar result was 


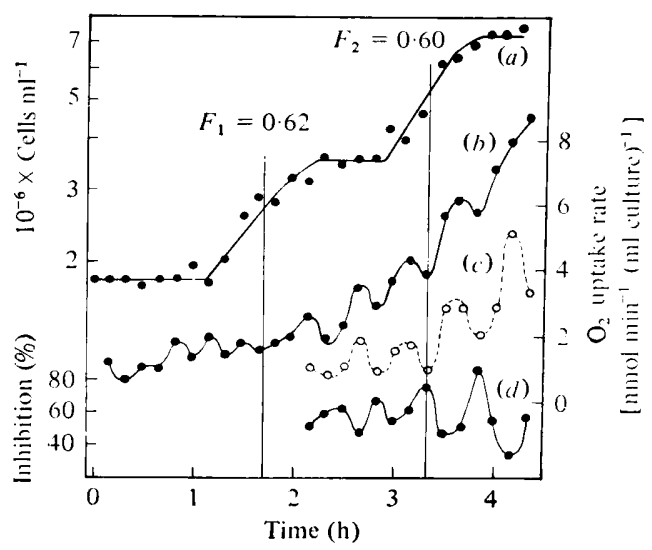

Fig. 3. Effect of DCCD on respiratory oscillations in a synchronously dividing culture of C. utilis with glucose as carbon source. The synchronous culture initially contained $10 \%$ of the original population of the exponentially growing culture. (a) Cell numbers; (b) respiration rate in the absence of DCCD; $(c)$ respiration rate in the presence of $75 \mu \mathrm{M}-\mathrm{DCCD} ;(d)$ inhibition of respiration $(\%)$ resulting from addition of DCCD.

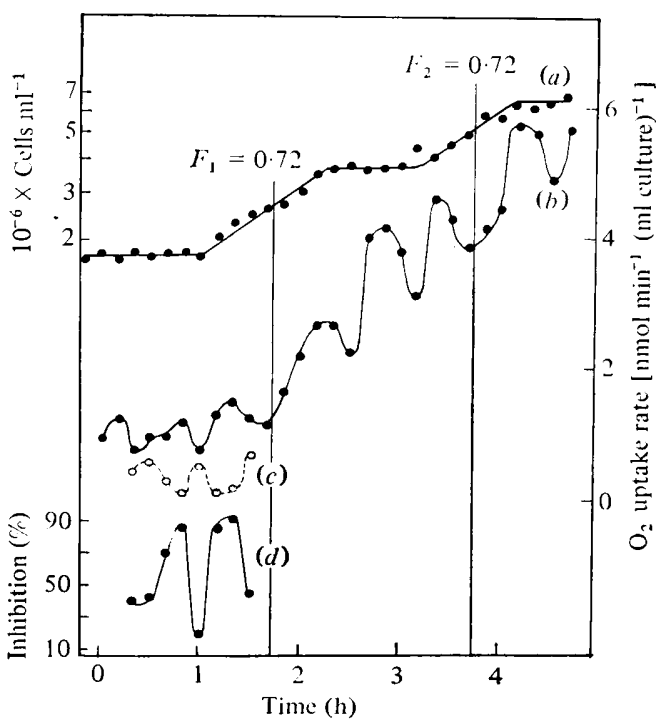

Fig. 4

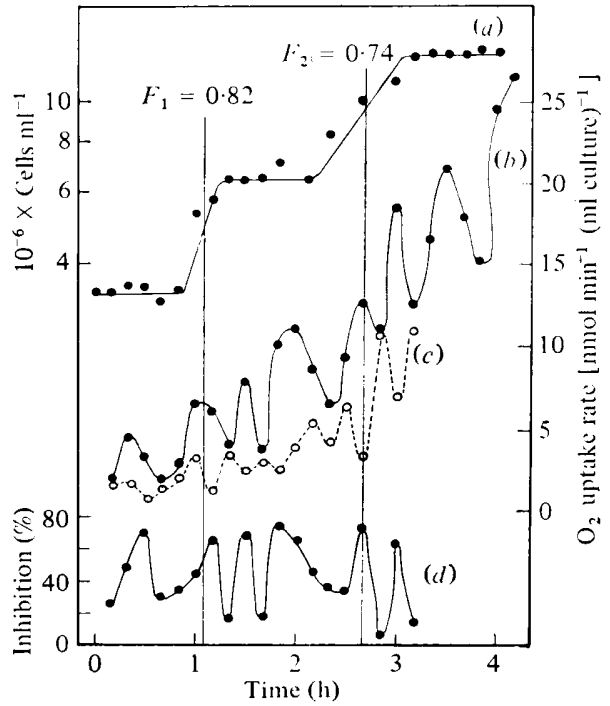

Fig. 5

Fig. 4. Effect of DCCD on respiratory oscillations in a synchronously dividing culture of $C$. utilis with acetate as carbon source. The synchronous culture initially contained $10 \%$ of the original population of the exponentially growing culture. (a) Cell numbers; $(b)$ respiration rate in the absence of DCCD; $(c)$ respiration rate in the presence of $30 \mu \mathrm{M}-\mathrm{DCCD} ;(d)$ inhibition of respiration $(\%)$ resulting from addition of DCCD.

Fig. 5. Effect of cyanide on respiratory oscillations in a synchronously dividing culture of $C$. utilis with glycerol as carbon source. The synchronous culture initially contained $10 \%$ of the original population of the exponentially growing culture. (a) Cell numbers; $(b)$ respiration rate in the absence of $\mathrm{KCN}$; (c) respiration rate in the presence of $50 \mu \mathrm{M}-\mathrm{KCN}$; $(d)$ inhibition of respiration (\%) resulting from the addition of $\mathrm{KCN}$. 
obtained previously with organisms synchronized by selection on a sucrose gradient (R. K. Poole \& D. Lloyd, cited by Lloyd, 1974).

The changes in respiration rate in a synchronous culture growing with acetate are shown in Fig. 4; the time between mid-points in cell doublings in this experiment was 120 min. The respiration rate increased exponentially overall but, again, superimposed on this pattern was an oscillation with a periodicity of about one-third of the cell cycle. Addition of $30 \mu \mathrm{M}-\mathrm{DCCD}$ to culture samples gave about $90 \%$ inhibition of respiration at a respiratory maximum, but only 25 to $40 \%$ inhibition at a respiratory minimum. Addition of $30 \mu \mathrm{M}$ $\mathrm{KCN}$ similarly produced a greater degree of inhibition at a respiratory maximum. These inhibitor effects were therefore quite different from those observed with synchronous cultures growing with glucose. Uncoupling of the reactions of energy conservation in acetate-grown cultures with $3 \mu \mathrm{M}$-CCCP gave between 30 and $50 \%$ stimulation of respiration at respiratory minima but produced no detectable acceleration of respiration at respiratory maxima (not shown).

In synchronous cultures growing with glycerol, the effects on respiration of inhibition of electron transport, energy transfer and uncoupling of energy conservation by KCN, DCCD and CCCP, respectively, were qualitatively similar to those observed for acetate-containing cultures. For the culture in Fig. 5, the mean period of the respiratory oscillation was $33 \mathrm{~min}$ and the peak-trough amplitude of the oscillation was between 22 and $100 \%$ of the minimum value. The overall increase was exponential and the respiration rate approximately doubled over each cell cycle $(95 \mathrm{~min})$. Maximum inhibition $(70 \%)$ by $50 \mu \mathrm{M}-\mathrm{KCN}$ observed at respiratory maxima contrasted with the low inhibition at respiratory minima (10 to $30 \%$ ). DCCD $(80 \mu \mathrm{M})$ also inhibited respiration preferentially at respiratory maxima. As with cells growing synchronously with glucose or acetate, CCCP $(3 \mu \mathrm{M})$ gave most marked uncoupling of respiration at respiratory minima.

\section{Heat evolution in synchronous cultures}

The rate of heat evolution by a culture growing synchronously with glycerol increased smoothly (and almost linearly) through three cycles of growth and division (Fig. 6). Respiration rates showed the usual oscillation with a period of about one-third of the cell cycle. Similar results were obtained with cultures growing synchronously with glucose or acetate; we could not detect any fluctuations in the rates of heat dissipation irrespective of the carbon source utilized.

\section{DISCUSSION}

Synchronous cultures of $C$. utilis established by continuous-flow centrifugal size selection show respiration oscillations. Comparison with the smoothly exponential increases in respiration of asynchronously dividing control cultures exposed to a higher centrifugal force than that employed for selection during establishment of synchrony confirms that these oscillations do not arise as a consequence of metabolic perturbation by the selection procedure employed. It has been noted, however, that relatively short periods of anaerobiosis (15 min or more) can induce respiratory oscillations which differ in two respects from the cell cycle-dependent oscillations observed here (Lloyd \& Ball, 1979): (i) their period is about $5 \mathrm{~min}$, and (ii) they are highly damped and are not detectable after $50 \mathrm{~min}$.

The cell-cycle dependent oscillations occur in cultures growing synchronously with either glucose, acetate or glycerol as sole carbon source. Thus $C$. utilis differs in this respect from Schizosaccharomyces pombe, which showed oscillations in respiration when grown with glucose (Poole et al., 1973) but not with glycerol (Poole \& Lloyd, 1974), and also from Saccharomyces cerevisiae, which showed respiratory oscillations in glucose-grown but not in maltose-grown synchronous cultures (Dharmalingam \& Jayaraman, 1973).

Table 1 summarizes the cell-cycle times and effects of KCN, DCCD and CCCP on C. utilis growing with the three different carbon sources. The response of acetate- and 


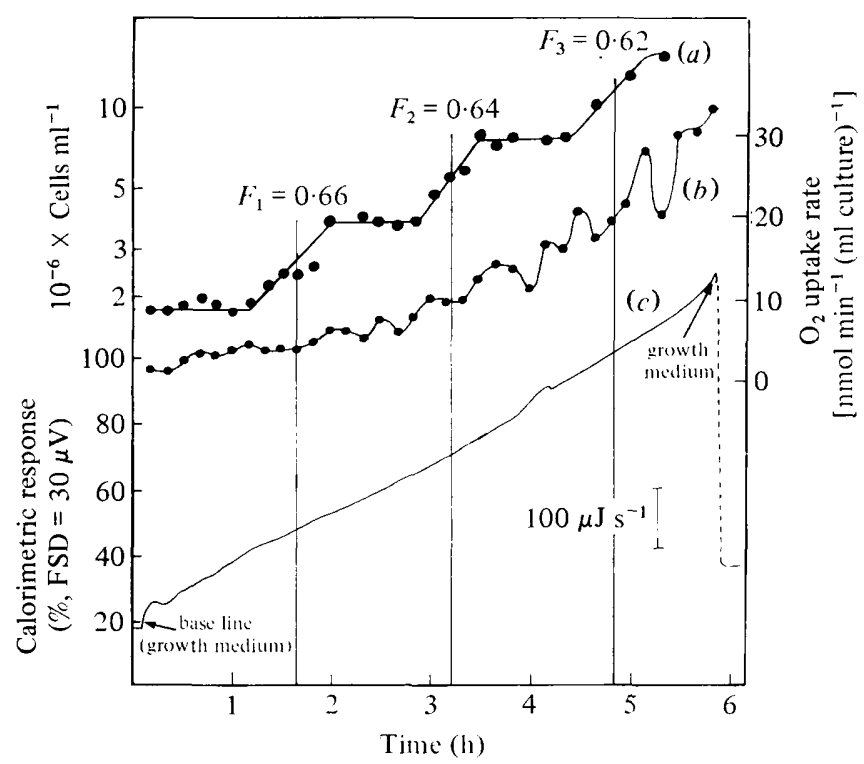

Fig. 6. Oxygen uptake and heat evolution of a synchronously dividing culture of $C$. utilis with glycerol as carbon source. The synchronous culture initially contained $10 \%$ of the original population of the exponentially growing culture. (a) Cell numbers; (b) respiration rate; $(c)$ heat evolution measured continuously using a flow microcalorimeter.

\section{Table 1. Cell cycle times and the effects of $K C N, D C C D$ and $C C C P$ on the respiration of synchronous cultures of C. utilis}

Cell cycle times (mean values \pm standard deviations, for the number of experiments shown in parentheses) were measured from the time of selection to the first mid-point of increase in cell numbers (first cycle), and from the first to second mid-points of increases in cell numbers (second cycle). + indicates maximum effect at respiratory maxima; - indicates maximum effect at respiratory minima.

\begin{tabular}{lcccccc} 
& \multicolumn{2}{c}{ Cell cycle time (min) } & & \multicolumn{3}{c}{ Effect of } \\
\cline { 2 - 4 } Carst cycle & Second cycle & & KCN & DCCD & CCCP \\
Glucose & $94 \cdot 6 \pm 17 \cdot 4(10)$ & $102 \cdot 8 \pm 12 \cdot 1(9)$ & - & - & - \\
Acetate & $110 \cdot 8 \pm 16 \cdot 4(12)$ & $118 \cdot 1 \pm 12 \cdot 1(11)$ & + & + & - \\
Glycerol & $91 \cdot 4 \pm 11 \cdot 3(9)$ & $98 \cdot 2 \pm 9 \cdot 5(7)$ & + & + & -
\end{tabular}

glycerol-grown cultures to the cytochrome oxidase and ATP synthetase inhibitors and to the uncoupler of energy conservation can be explained on the basis that the respiratory oscillation reflects in vivo mitochondrial respiratory control (Chance \& Williams, 1956), as was shown to be the case in A. castellanii (Edwards \& Lloyd, 1978). Thus the rate of respiration is primarily determined by the energetic requirements of biosynthetic reactions. The effect of CCCP on glucose-grown cultures is also consistent with this explanation, but the effects of KCN and DCCD on such cultures are anomalous. Secondary interactions between the products of mitochondrial energy conservation and the initial reactions of glucose catabolism via feedback loops and allosteric control sites complicate the interpretation of the effects of mitochondrial inhibitors on the respiration of glucose in growing organisms (see also Poole et al., 1973). No fluctuations in heat evolution could be detected by flow microcalorimetric monitoring of synchronous $C$. utilis cultures: oscillations of heat evolution have been detected in synchronous cultures of $T$. pyriformis (Lloyd et al., 1978). That heat dissipation did not parallel either growth or respiration was remarkable, 
but a similar observation was made with $S$. pombe (Poole et al., 1973) and further work is necessary to quantify these relationships.

The period of the respiratory oscillations was similar in cultures growing synchronously with any of the three carbon sources. Whereas the respiratory oscillations previously described in Crithidia fasciculata (Edwards et al., 1975), T. pyriformis (Lloyd et al., 1978) and $A$. castellanii (Edwards \& Lloyd, 1978) all showed periods of about $1 \mathrm{~h}$ at $30^{\circ} \mathrm{C}$, those in C. utilis were of a frequency of about $2 \mathrm{~h}^{-1}$ at the same temperature.

The authors thank Mrs L. John and Miss J. Ball for expert technical assistance. J. K. received a Staff Training Scheme Grant from Universiti Kebangsaan Malaysia.

\section{REFERENCES}

Blumenthal, L. K. \& ZahleR, S. A. (1962). Index for measurement of synchronization of cell populations. Science 135, 724.

Chagla, A. H. \& Griffiths, A. J. (1978). Synchronous cultures of Acanthamoeba castellanii and their use in the study of encystation. Journal of General Microbiology 108, 39-43.

Chance, B. \& Williams, G. R. (1956). The respiratory chain and oxidative phosphorylation. Advances in Enzymology 17, 65-134.

Dharmalingam, K. \& Jayaraman, J. (1973). Mitochondriogenesis in synchronous cultures of yeast. I. Oscillatory patterns of respiration. Archives of Biochemistry and Biophysics 157, 197-202.

EdwARds, S. W. \& LloYd, D. (1978). Oscillations of respiration and adenine nucleotides in synchronous cultures of Acarthamoeba castellanii: mitochondrial respiratory control in vivo. Journal of General Microbiology 108, 197-204.

Edwards, C., Statham, M. \& Lloyd, D. (1975). The preparation of large-scale synchronous cultures of the trypanosomatid, Crithidia fasciculata, by cell-size selection: changes in respiration and adenylate charge through the cell-cycle. Journal of General Microbiology 88, 141-152.

Kováč, L., Galeottı, T. \& Hess, B. (1968). Oligomycin-like inhibition of yeast respiration by $N, N^{\prime}$ dicyclohexylcarbodi-imide, and the nature of energy-coupling in intact yeast cells. Biochimica et biophysica acta 153, 715-717.

Lloyd, D. (1974). The Mitochondria of Microorganisms. London: Academic Press.
Lloyd, D. \& BALl, J. (1979). Perturbation of respiration in Candida utilis: induction of metabolic oscillations. Journal of General Microbiology 114, 463-466.

Lloyd, D. \& Brookman, J. S. G. (1967). An oxygen electrode reaction vessel. Biotechnology and Bioengineering 9, 271-272.

Lloyd, D., John, L., Edwards, C. \& Chagla, A. H. (1975). Synchronous cultures of microorganisms: large-scale preparation by continuousflow size selection. Journal of General Microbiology 88, 153-158.

Lloyd, D., Phillips, C. A. \& Statham, M. (1978). Oscillations of respiration, adenine nucleotide levels and heat evolution in synchronous cultures of Tetrahymena pyriformis ST prepared by continuous-flow selection. Journal of General Microbiology 106, 19-26.

Poole, R. K. \& LloYd, D. (1974). Changes in respiratory activities during the cell cycle of the fission yeast Schizosaccharomyces pombe $972 h^{-}$ growing in the presence of glycerol. Biochemical Journal 144, 141-148.

Poole, R. K., Lloyd, D. \& Kemp, R. B. (1973). Respiratory oscillations and heat evolution in synchronously dividing cultures of the fission yeast Schizosaccharomyces pombe $972 \mathrm{~h}^{-}$. Journal of General Microbiology 77, 209-220.

Williamson, D. H. \& Scopes, A. W. (1960). The behaviour of nucleic acids in synchronously dividing cultures of Saccharomyces cerevisiae. Experimental Cell Research 20, 338-349. 